\title{
TAGUNG
}

\section{Nationale Parlamente in der Europäischen Union: Demokratie im Mehrebenensystem der EU}

\author{
Claudia Hefftler und Linda Dieke*
}

Fragen der demokratischen Legitimation europäischer Entscheidungen haben mit den Reformen des Vertrags von Lissabon, aber auch mit Ausbruch der Wirtschafts- und Finanzkrise, an Aktualität gewonnen. Wie nationale Parlamente auf diese neuen Herausforderungen reagieren und welche Möglichkeiten sie haben, ihre Regierungen im europäischen Mehrebenensystem zu kontrollieren, stand im Mittelpunkt der zweitägigen Konferenz des Projekts „Observatory of Parliaments after the Lisbon Treaty“ (OPAL) $)^{1}$ der Universität zu Köln und des Arbeitskreises Europäische Integration e.V. Teilnehmer aus Wissenschaft und Praxis trafen hier aus ganz Europa zusammen, um die neuesten Forschungsergebnisse zur Rolle nationaler Parlamente im politischen System der Europäischen Union zu präsentieren und zu diskutieren. In sechs Panels und vier Workshops mit unterschiedlichen Themenschwerpunkten nahmen Experten aus fast allen EU-Mitgliedstaaten an dem erkenntnisreichen Informationsaustausch und den intensiven Diskussionen teil. Ergebnisse werden 2014 zum einen im Special Issue der West European Politics und zum anderen im „Palgrave Handbook on National Parliaments and the EU“ veröffentlicht.

\section{Forschungsperspektiven auf nationale}

Parlamente in EU-Angelegenheiten

Nach der Begrüßung durch Katjana Gattermann, Thomas Christiansen, Claudia Hefftler und Olivier Rozenberg ging es um neue For-

\section{National Parliaments in the EU: The \\ Performance of Multilevel Democracy in Europe}

Konferenz des OPAL Projekts (Universität zu Köln) und des Arbeitskreises Europäische Integration e.V.

Mit finanzieller Unterstützung der Europäischen Kommission und der Deutschen Forschungsgemeinschaft

7.-8. März 2013, Berlin

\section{Welcome}

Katjana GATTERMANN, Thomas CHRISTIANSEN, Claudia HEFFTLER and Olivier ROZENBERG

Research on national parliaments: Which way forward?

Chair

Thomas CHRISTIANSEN

National Parliaments after Lisbon: Towards the Mainstreaming of EU Affairs?

Katjana GATTERMANN, Anna-Lena HÖGENAUER and Ariella HUFF

Measuring National Parliaments' level of influence in EU decision making - the results from a latent structure analysis of EU 27

Mads Dagnis JENSEN and Dorte MARTINSEN

Discussant

Davor JANCIC

* Claudia Hefftler, Wissenschaftliche Mitarbeiterin, Jean Monnet Lehrstuhl - Prof. Dr. Wolfgang Wessels, Universität zu Köln.

Linda Dieke, Studentische Hilfskraft, Jean Monnet Lehrstuhl - Prof. Dr. Wolfgang Wessels, Universität zu Köln.

1 Internetseite des OPAL-Projekts: http://www.opal-europe.org/. 
schungsansätze und Perspektiven zu nationalen Parlamenten.

Katjana Gattermann, Anna-Lena Högenauer und Ariella Huff stellten fest, dass in den letzten Jahren ein ,Mainstreaming' von EU-Themen in nationalen Parlamenten zu beobachten gewesen sei. Es sei als neuer Trend der Europäisierung zu verstehen, dass Parlamente EUAngelegenheiten in den Fachausschüssen behandeln und nicht in einem gesonderten EUAusschuss. Bei dieser Entwicklung handele es sich um eine Reaktion auf den wachsenden Druck der europäischen Integration. Indikatoren, anhand derer das ,Mainstreaming von EU-Themen in nationalen Parlamenten gemessen werden könnte, seien der Zuwachs an Fachausschüssen, die EU-Themen behandeln, die Erhöhung der Anzahl der Referenten in diesen Ausschüssen, verstärkte interparlamentarische Aktivitäten sowie eine steigende Anzahl an Plenardebatten zu EU-Themen.

Mads Dagnis Jensen diskutierte die Frage, wie die Einflussstärke von Parlamenten auf EUAngelegenheiten gemessen werden kann. Bislang gebe es hierfür noch kein allgemein anerkanntes Konzept für die Messung der Stärke von Parlamenten. Aus der Forschungsliteratur griff Jensen als die drei wichtigsten Faktoren den Zugang zu Information, die Durchsetzungskraft gegenüber der Regierung sowie die Aufsichtsfunktionen des Parlaments auf. Zur Überprüfung dieser Annahme hat er eine multivariate Analyse durchgeführt, die auf Daten aus einer Online-Befragung sowie mehreren Berichten der Conference of Parliamentary Committees for Union Affairs of Parliaments of the European Union (COSAC) basiert.

Davor Jancic sprach sich in seinem Kommentar dafür aus, dass in der Tat eine neue Phase der Europäisierung zu beobachten sei, es aber große Varianz gebe. Außerdem sei die Salienz europäischer Angelegenheiten ein wichtiger Faktor in allen Kontrollsystemen nationaler Parlamente, die aber mit bisherigen Mitteln noch nicht wissenschaftlich erfasst werden könne.
National Parliaments and the Sovereign Debt

Crisis

Chair

Katjana GATTERMANN

De-Parliamentarisation or new opportunities for involvement: First and second class parliaments in the financial and debt crisis?

Katrin AUEL and Oliver HÖING

Are we all austerians now? An analysis of parliamentary parties' positioning on anti-crisis measures

Aleksandra MAATSCH

Discussant

Julie SMITH

Inter-parliamentary relations in the $\mathbf{E U}$

Chair

Thomas CHRISTIANSEN

The legal and living constitution of multilevel parliamentarism after Lisbon

Andreas MAURER

New players in the EU political system? Incentives and strategies of national parliaments beyond the domestic arena

Katjana GATTERMANN and Claudia

HEFFTLER

"We want to keep it light and flexible" - National Parliaments and the European Parliament in the Inter-parliamentary Conference on CFSP/CSDP Jan WOUTERS and Kolja RAUBE

Discussant

Eric MIKLIN

Parliamentary scrutiny of EU affairs

Chair

Oliver HÖING

The Role of National Parliaments in Scrutinizing EU Foreign Policy

Ariella HUFF und Julie SMITH

Parliamentary scrutiny of EU affairs after the Lisbon Treaty: (missing) factors of improvement

Alexander STRELKOV

Fighting Back? EU Affairs in National Legislatures Katrin AUEL, Olivier ROZENBERG and Angela TACEA

Discussant

Tom DELREUX 
Nationale Parlamente und die Staatsschuldenkrise

Im zweiten Konferenzpanel wurde thematisiert, wie die aktuelle Finanz- und Schuldenkrise die Aktivitäten nationaler Parlamente und das Verhalten der Parteien beeinflusst. Dazu wurden zwei länderübergreifende komparative Forschungsansätze vorgestellt.

Katrin Auel und Oliver Höing präsentierten einen neuen Index zur Messung der parlamentarischen Einbindung in Krisenpolitik anhand der Auswertungen von Aktivitäten der Parlamente. Auel und Höing entdeckten eine erhebliche Variation zwischen verschiedenen Staaten und stellten einige Hypothesen auf, mit deren Hilfe die Varianz erklärt werden kann. Als Variablen wurden unter anderem die generelle Stärke des Parlaments gegenüber seiner Exekutive, die Existenz euroskeptischer Parteien sowie das Ausmaß, in dem ein Land von der Eurokrise betroffen ist, vorgestellt. Die ausgewerteten Daten aus 30 Parlamentskammern von 2010 bis 2012 konnten die Hypothesen noch nicht bestätigen, verdeutlichten aber den noch notwendigen Forschungsbedarf.

Aleksandra Maatsch beschäftigte sich in ihrem Beitrag mit der Frage, ob und in welchem Aus$\mathrm{maß}$ die Krise die ideologische Grundhaltung der Parteien auf dem links-rechts Spektrum in den Parlamenten acht europäischer Staaten berührt. Um zu untersuchen, ob die Parteien bei ihren traditionellen Positionen blieben oder sich ihre ideologische Einstellung zu marktwirtschaftlicher Liberalisierung im Zuge der Krise veränderte, wurde insbesondere berücksichtigt, wie der betreffende Staat von der Eurokrise betroffen ist. Grundlage der Untersuchung waren Daten über Plenardebatten zur Europäischen Finanzstabilisierungsfazilität (EFSF). Die Ergebnisse wiesen nach Maatsch darauf hin, dass Parteipositionen in den Kreditgeberländern eher ihren traditionellen Positionen entsprachen. Dahingegen befürworteten Parteien in den Schuldnerstaaten eher ein keynesianisches Anti-Krisen-Management, unabhängig von ihrer politischen Grundhal-
Inter-parliamentary co-ordination in the $\mathbf{E U}$

Chair

Anna-Lena HÖGENAUER

The role of national parliaments in the policing of the subsidiarity principle

Katarzyna GRANAT

The Early Warning system in motion: comparing different practices

Gracia Vara ARRIBAS

The role of non-political actors in the

Europeanization of parliamentary affairs

Chair

Ariella HUFF

Much Ado About Nothing? Parliamentary Scrutiny in EU Affairs from a Governmental perspective

Katrin AUEL, Renaud DEHOUSSE, Olivier ROZENBERG, Angela TACEA and Anja THOMAS

Administrators "precooking" European affairs? The role of civil servants within national parliaments after the Lisbon Treaty

Christine NEUHOLD and Anna-Lena

HÖGENAUER

Discussant

Andreas MAURER

Handbook Panel 1: Mandating functions of parliaments

Chair

Julie SMITH

Austria (Eric MIKLIN), Denmark (Mette Buskjær CHRISTENSEN), Latvia (Jãnis IKSTENS), Estonia (Piret EHIN), Slovenia (Sabina LANGE), Sweden (Hans HEGELAND)

Handbook Panel 2: Parliaments in the new member states

Chair

Olivier ROZENBERG

Bulgaria (Dobrin KANEV), Croatia (Hrvoje BUTKOVIĆ), Czech Republic (Alexander STRELKOV and Martin HRABÁLEK), Lithuania (Jūratė NOVAGROCKIENĖ), Malta (Roderick PACE), Romania (Angela TACEA)

Handbook Panel 3: Varieties of parliamentary scrutiny

Chair

Christine NEUHOLD 
tung und ihrer Zugehörigkeit zu Regierung oder Opposition.

\section{Interparlamentarische Beziehungen in der EU}

Ein weiteres Panel diente der Diskussion von Chancen und Herausforderungen der interparlamentarischen Kooperation zwischen nationalen Parlamenten sowie mit dem Europäischen Parlament.

Andreas Maurer eröffnete seinen Beitrag mit einer kritischen Bewertung der Beziehung zwischen nationalen Parlamenten und dem Europäischen Parlament. Es sei nicht anzunehmen, dass durch eine verstärkte interparlamentarische Zusammenarbeit die parlamentarische Kontrolle innerhalb der Europäischen Union gestärkt werden kann, die das Demokratiedefizit der Europäischen Union verringern würde. Er begründete seine Zweifel damit, dass das Europäische Parlament durch die Kooperation andere Ziele verfolge als die nationalen Parlamente. Zudem werfe ein Mangel an verbindlichen Entscheidungen auf interparlamentarischen Treffen die Fragen nach der Effektivität dieses Instruments und einem Legitimitätsgewinn auf.

Jan Wouters präsentierte Ergebnisse zu der Beziehung zwischen dem Europäischen und den nationalen Parlamenten in der Gemeinsamen Außen- und Sicherheitspolitik (GASP) und der Gemeinsamen Sicherheits- und Verteidigungspolitik (GSVP) und legte einen speziellen Fokus auf die diesbezügliche Interparlamentarische Konferenz. Es gebe drei Hauptmotive für parlamentarische Akteure zur Kooperation: Informationsaustausch, die Stärkung interparlamentarischer Netzwerke sowie parlamentarische Kontrolle in diesem von der Exekutive dominierten Bereich. Die Forschungsergebnisse zeigten, dass die Interparlamentarische Konferenz vor allem als Instrument zum Informationsaustausch angesehen werde. Hierfür sind laut Wouters Probleme, wie etwa der nicht rechtlich bindende Charakter der verabschiedeten Resolutionen, verantwortlich. Nichtsdestotrotz sei die Erforschung
Belgium (Tom DELREUX and François RANDOUR), France (Olivier ROZENBERG, Angela TACEA and Anja THOMAS), Germany (Oliver HÖING), Luxembourg (Astrid SPREITZER), Netherlands (Anna-Lena HÖGENAUER), Poland (Jan BARCZ and Anna PUDLO), UK (Julie SMITH and Ariella HUFF)

Handbook Panel 4: Parliaments in times of crisis Chair

Claudia HEFFTLER

Cyprus (Kalliope AGAPIOU-JOSEPHIDES), Greece (Dimitris SOTIROPOULOS), Ireland (Gavin BARRETT), Italy (Sabrina CAVATORTO), Portugal (Davor JANCIC), Spain (Mario KÖLLING and Ignacio MOLINA)

Expert table: Perspectives for Multilevel Democracy in the European Union

Chair

Katrin AUEL

Thomas CHRISTIANSEN, Enikő GYÖRI, Hans HEGELAND, Haris KOUNTOUROS, Andreas MAURER and Lord John ROPER

der interparlamentarischen Zusammenarbeit wichtig für das Verständnis künftiger Entwicklungen, da sich gerade im Bereich der GASP/ GSVP die Kooperation intensiviere.

Katjana Gattermann und Claudia Hefftler untersuchten Gründe für das unterschiedliche Engagement nationaler Parlamente im politischen Dialog mit der Kommission und dem sogenannten Frühwarnmechanismus. Ihre multivariate Regressionsanalyse lieferte interessante Ergebnisse: Der Einfluss der Ressourcenausstattung eines Parlaments, seiner Informationsrechte und der Rolle der Europaausschüsse auf die Anzahl der (begründeten) Stellungnahmen war nicht eindeutig feststellbar. Jedoch konnte gezeigt werden, dass interparlamentarische Zusammenarbeit sowie politische Kontroversität Einfluss auf die Anzahl der begründeten Stellungnahmen in einem Mitgliedstaat haben. Gattermann und Hefftler sprachen sich daher für eine Vertiefung der Forschung in diesem Bereich aus. 
Parlamentarische Kontrolle von EU-Angelegenheiten

Die unterschiedlichen Aspekte der Kontrolle europäischer Angelegenheiten standen im Fokus des nachfolgenden Veranstaltungsteils.

Ariella Huff und Julie Smith sprachen sich für eine spezifische Untersuchung der Kontrolle in der GASP aus, wo die nationalen Parlamente bislang und auch seit dem Inkrafttreten des Vertrags von Lissabon eine eher schwache Stellung haben. Sie bedienten sich der stark gekoppelten Konzepte von ,authority, ability, and attitude", um die Macht der Parlamente in diesem Politikfeld zu charakterisieren. Sie zeigten, dass der Grad der Kontrolle nicht nur von formalen Rechten gegenüber der Regierung, sondern auch von der Motivation der Parlamentarier abhängt, diese Rechte auszuspielen.

Alexander Strelkov untersuchte die Gründe für die fortdauernden Unterschiede zwischen den Kontrollmechanismen der Parlamente. Als erklärende Faktoren nannte er die Beteiligung von spezialisierten Ausschüssen und den Einfluss nationaler Parteien. Aus seinen auf Basis eines qualitativen Forschungsdesigns gewonnen Ergebnissen zu den Parlamenten Schwedens, Tschechiens und Rumäniens konnte er ableiten, dass die nationalen Parlamente ihre Kontrollmöglichkeiten zwar nutzen, aber auf eingeschränkte Weise: Sie überwachen mögliche Kompetenzüberschreitungen der Europäischen Union, nehmen jedoch keinen inhaltlichen Einfluss auf die Politikgestaltung.

Einen neuen Indikator für die Messung der Kontrolle nationaler Parlamente entwickelten Katrin Auel und Angela Tacea. Dazu verwenden sie zwei Variablen: die formellen Beteiligungsrechte und die tatsächlichen Aktivitäten von Parlamenten. Die Daten entnahmen sie den OPAL-Länderberichten, dem 17. COSAC-Bericht, der Studie „Democratic Control in the
Member States of the European Council and the Euro zone summits" ${ }^{\text {"2 }}$ und der quantitativen Datensammlung des Projekts OPAL für den Zeitraum von 2010 bis 2012. Erste Ergebnisse ihrer Forschung bestätigten die Korrelation zwischen den formellen Beteiligungsrechten und den tatsächlichen Aktivitäten, obwohl auch Ausnahmen zu beobachten sind, wie im Fall des Deutschen Bundestags, der seine starken Beteiligungsrechte nur bedingt nutzt.

\section{Interparlamentarische Kooperation in der EU}

Im fünften Themenbereich ging es um den Frühwarnmechanismus, also die Möglichkeit zur Abgabe einer begründeten Stellungnahme eines nationalen Parlaments zu einem Gesetzesvorschlag der Kommission. Von den Teilnehmern wurde diskutiert, welche Faktoren zur Beteiligung der Parlamente im Frühwarnmechanismus beitragen und wie sich regionale Parlamente einbringen.

Katarzyna Granat beschäftigte sich in ihrem Beitrag mit der Frage, warum Parlamente an der Subsidiaritätsprüfung teilnehmen. Sie identifizierte bestimmte Faktoren, darunter verfassungsbedingte Einwände, besondere nationale Interessen oder den redistributiven Charakter von Kommissionsvorschlägen. Granat untersuchte außerdem, wie sich parlamentarische Akteure im Prozess der Subsidiaritätsprüfung positionieren. In der Regel war dabei eine Übereinstimmung von Regierungs- und Oppositionsparteien zu vermerken, wobei es gerade in Deutschland - auch Ausnahmen gebe.

Die gesonderte Rolle der regionalen Parlamente war Thema von Gracia Vara Arribas, die die unterschiedliche Herangehensweise dieser Parlamente an die durch den Vertrag von Lissabon erweiterten Möglichkeiten der Beteiligung hervorhob. Vara Arribas erläuterte auch Schwierigkeiten für nationale Parlamente, wie etwa die kurze Frist von acht Wochen zur Ab-

2 Wolfgang Wessels/Olivier Rozenberg/Mirte van den Berge/Claudia Hefftler/Valentin Kreilinger/Laura Ventura: The democratic control in the Member States of the European Council and the Euro zone summits, European Parliament, Directorate-General for Internal Policies: Study 2013. 
gabe einer begründeten Stellungnahme, die eine stärkere Beteiligung verhinderten. Insgesamt sei der Einfluss der Regionalparlamente trotz der neuen Regelung immer noch limitiert.

\section{Die Rolle nichtparlamentarischer Akteure}

Das sechste Panel bot Raum für die Analyse des Einflusses nichtparlamentarischer Akteure auf parlamentarische Aktivitäten zu EU-Themen.

Olivier Rozenberg et al. kennzeichneten in ihrem Vortrag die Minister als Hauptakteure in europäischen Angelegenheiten und untersuchten, inwiefern parlamentarische Aktivitäten die Handlungen der Minister beeinflussten. $\mathrm{Zu}$ diesem Zweck entwickelten sie unter anderem eine Kategorisierung in drei Typen von Ministern und analysierten die Aktivität letzterer in Plenardebatten, im Ausschuss für europäische Angelegenheiten und im Ministerrat. Die Auswertung ergab, dass Minister teilweise ihr Parlament benutzten, um ihre Position zu stärken, teilweise auch die Beschlüsse der Parlamente vorformulierten.

Mit der Parlamentsverwaltung und deren Einfluss auf die Kontrolle von EU-Angelegenheiten befassten sich Christine Neuhold und Anna-Lena Högenauer. Mit dem Vertrag von Lissabon und der Einführung des Frühwarnmechanismus habe es einen erhöhten Druck auf die Verwaltungen der Parlamente gegeben. Eine Analyse von Umfragedaten und Interviewmaterial zu acht Parlamenten bestätigte ihre Hypothese, dass der Vertrag von Lissabon die Rolle der Bürokraten gestärkt habe. Dies ergebe sich insbesondere durch den Informationsvorsprung und die Möglichkeit des AgendaSettings der Verwaltung. Neuhold und Högenauer wiesen aber auch darauf hin, dass die endgültigen Entscheidungen immer noch bei den gewählten Abgeordneten lägen.

\section{Länderberichte zu parlamentarischer Kontrolle in EU-Angelegenheiten}

Parallel zu den regulären Panels wurden auf der Konferenz Beiträge für das „Palgrave
Handbook on National Parliaments and the EU“ vorgestellt. Autoren aus nahezu allen EUMitgliedstaaten präsentierten ihre Forschung zu den jeweiligen nationalen Parlamenten, wie sie in die einzelnen Länderkapitel des Handbuchs einfließt.

In vier Panels wurden die Themen der parlamentarischen Regierungsmandate, Parlamentsaktivitäten in den neuen Mitgliedstaaten, Unterschiede der Kontrollsysteme in Zweikammerparlamenten und Lage der Parlamente in den Krisenstaaten behandelt.

Der Vergleich zwischen den Staaten mit Mandatsrechten, wie Dänemark, Österreich, Lettland, Estland, Slovenien, Finnland und Schweden, zeigte, dass allgemein eine große Diskrepanz zwischen formalen Rechten und ihrer Ausübung besteht. Selbst im Lehrbuch-Fall der parlamentarischen Regierungsmandate, Dänemark, zeigen sich Schattenseiten eines Mandat-Systems. EU-Angelegenheiten werden hier diskret hinter verschlossenen Türen mit der Regierung ausgehandelt. Dabei sind nur wenige auf die Europäische Union spezialisierte Parlamentarier involviert. Somit werden EU-Themen nicht in die Öffentlichkeit getragen und das Parlament erfüllt die Funktion der öffentlichen Legitimation einer politischen Entscheidung nur bedingt.

Die in 2004 beigetretenen Mitgliedstaaten konnten von den Erfahrungen anderer Parlamente profitieren, wobei einige sich am Mandatsmodell Dänemarks orientierten. Aufgrund der Dominanz der Exekutive in einigen Staaten ist der Einfluss der Parlamente trotz einer formal starken Position in der politischen Praxis häufig beschränkt.

In der Analyse der Zweikammerparlamente ist die Frage nach der unterschiedlichen Einflussstärke der zwei Kammern diskutiert worden. Im Bereich der EU-Angelegenheiten, insbesondere des Frühwarnmechanismus, zeigt sich, dass zweite Kammern häufig sehr engagiert sind. 
Mit Blick auf die Parlamente in den Krisenstaaten, wie Zypern, Spanien, Griechenland, Italien, Irland und Portugal, fiel auf, dass die meisten unter der dominanten Position der Exekutive litten. Nicht nur der geringe Einfluss auf die Entscheidungen der Regierung lässt an der legitimatorischen Funktion der gewählten Repräsentanten zweifeln, sondern auch die nur bedingte Widerspiegelung der öffentlichen Meinung im Parlament. Eine eurokritische Haltung der Bevölkerung überträgt sich hier nur bedingt auf die Positionen und Wahlprogramme der politischen Parteien.

Externe Ereignisse, wie die Finanzkrise oder im Falle Deutschlands die Urteile des Bundesverfassungsgerichts, haben in einigen Parlamenten zu einem erhöhten Engagement zu EUThemen geführt.

Die Vorträge zu den Parlamenten beinahe aller EU-Mitgliedstaaten haben ein facettenreiches Bild der parlamentarischen Kontrollmöglichkeiten in der Europäischen Union und ihrer Grenzen in der politischen Praxis gezeichnet.

\section{Perspektiven für die Mehrebenendemokratie in der Europäischen Union}

Zum Abschluss der Konferenz kamen an einem Expertentisch zum Thema „Perspectives for Multilevel Democracy in the European Union“ Fachleute aus Wissenschaft und Praxis zusammen. Die Teilnehmer konstatierten, dass die Parlamente sich in der Krise sehr unterschiedlich entwickelt hätten. Während die Rolle einiger gestärkt worden sei, hätten andere noch nicht die richtigen Mittel gefunden, um mit der
Krise umzugehen. Im Ergebnis sei aber eine wachsende direkte Beteiligung der Staats- und Regierungschefs sowie der europäischen Wirtschaftsminister zu beobachten, wodurch auch Abgeordnete sich zunehmend mit EU-Themen auseinandersetzten. Einigkeit herrschte auch darüber, dass die demokratische Legitimität der Mehrebenendemokratie auf der Ebene der nationalen Parlamente und auf der Ebene des Europäischen Parlaments gesichert werden müsse. Bislang sei aber das Zusammenwirken nicht optimal, wenn etwa an den vom Europäischen Parlament organisierten interparlamentarischen Treffen aus einigen Staaten keine Vertreter teilnähmen. Dabei könnte das Europäische Parlament, etwa durch Unterstützung des Informationsaustauschs, durchaus in der Lage sein, die Rolle der nationalen Parlamente zu stärken. Der durch den Vertrag von Lissabon etablierte Frühwarnmechanismus wurde von den Experten kritisch beurteilt. Da die nationalen Parlamente kein starkes Interesse an rechtlicher Subsidiaritätskontrolle hätten, sondern eher an inhaltlichen Aspekten, versprachen sie sich keine lange Überlebensdauer des Frühwarnmechanismus in der Praxis.

Die Teilnehmer zeigten sich insgesamt sehr interessiert an den Ergebnissen der Konferenz und der Forschung des OPAL-Teams. Es herrschte Übereinstimmung darüber, dass nationale Parlamente in Zukunft eine wichtige Rolle in der Mehrebenendemokratie einnehmen werden und ihre unterschiedlichen Beteiligungen weiterhin Gegenstand der Forschung sein sollten. 